\title{
Risk of tuberculosis in patients treated with biological medicines
}

\author{
Homolka J \\ 1st Department of Tuberculosis and Respiratory Diseases, 1st Faculty of Medicine, Charles University, \\ Prague, Czech Republic. jhomolka@cesnet.cz
}

\begin{abstract}
OBJECTIVE: The number of medicines used for biological therapy is constantly increasing. Biologic medicines selectively block the immunological processes leading to autoimmune manifestations with tissue damage or block the progression of haemato-oncological or other malignancies. Biological medicines are usually monoclonal antibodies or inhibitors of certain cytokines, cell receptors and enzymes. Their administration results in immunosuppression associated with increased risk of infectious complications, including tuberculosis. Assessment of the risk of tuberculosis associated with biological medicines is an important part of the medical decision done by pneumologist. We describe the risks of different biologicals and suggest the possible necessary steps in evaluating the risk of tuberculosis in individual patient (Ref. 18). Text in PDF www.elis.sk.

KEY WORDS: tuberculosis, latent tuberculosis infection, biological medicine, screening, chemoprevention.
\end{abstract}

\section{Introduction}

Currently, the number of medicines used for biological therapy is constantly increasing. Up to $70 \%$ of newly developed medicines are called biologics. Over time, the number of medicines in this area is growing and so-called biosimilars are available in addition to the original medicines. Biological therapy is used as advanced therapy of certain cancer or inflammatory diseases. Biologic agents selectively block immunological processes leading to autoimmune manifestations with tissue damage or block the progression of haemato-oncological or other malignancies. Blocking the T- and Blymphocyte activation or blockade of biological effects of some cytokines are targeted treatments with a significant effect on clinical manifestations in haematological malignancies, rheumatic diseases, multiple sclerosis, Crohn's disease and other pathologies $(12,13)$.

Biological medicines are usually monoclonal antibodies or inhibitors of certain cytokines, cell receptors and enzymes. Their administration results in immunosuppression associated with increased risk of infectious complications, including tuberculosis. Assessment of the risk of tuberculosis associated with biological medicines cannot be based on the results of controlled clinical trials because these studies are relatively short-term, do not involve patients with multiple comorbidities and are primarily aimed at demonstrating the effect of the selected medicine.

Of the monoclonal antibodies, antibodies against CD20 (rituximab), anti-CD52 (alemtuzumab), anti-CD80 and CD86 (abatacept), anti-tumour necrosis factor- $\alpha$ (infliximab, etanercept, adalimumab, golimumab, certolizumab pegol), against the receptor for

1st Department of Tuberculosis and Respiratory Diseases, 1st Faculty of Medicine, Charles University, Prague, Czech Republic

Address for correspondence: J. Homolka, 1st Department of Tuberculosis and Respiratory Diseases, 1 st Faculty of Medicine, Charles University, Karlovo namesti 32, CZ-121 11 Prague 2, Czech Republic. interleukin-6 (tocilizumab), against the receptor for interleukin-1 (anakinra), IL-12, and IL-23 (ustekinumab) are associated with a potential risk of tuberculosis.

\section{Anti CD20 treatment}

Antigen on B-lymphocytes CD20 is an activated glycosylated phosphoprotein expressed on the surface of B-lymphocytes from pro-B phase $(\mathrm{CD} 45 \mathrm{R}+, \mathrm{CD} 117+)$, the concentration of which increases with maturation of B-lymphocytes. In humans, CD20 is encoded by MS4A1 gene located on chromosome 11 (11q12). This gene encodes the surface molecule on B-lymphocytes, which is important for development and differentiation of B-lymphocytes into plasma cells. A natural ligand for CD 20 is not known yet. This phospho-protein enables optimal immune response of Blymphocytes in particular against antigens independent on $\mathrm{T}$ lymphocytes. CD20 appears to act as a calcium channel in the cell membrane of B-lymphocytes. CD20 is expressed on the surface of B-lymphocytes throughout their development except for the earliest stage and development of plasma cells. CD20 is also expressed on the surface of cells in B-cell lymphomas, in B-cell chronic lymphocytic leukaemia, hairy-cell leukaemia and melanoma. CD20 positive cells were occasionally found in Hodgkin's disease, myeloma or thymoma.

CD20 is the binding site of monoclonal antibodies (rituximab) used in the treatment of B-cell lymphomas and leukaemias. Rituximab is also used as maintenance therapy of ANCA-associated vasculitis $(8,10,14)$. The risk of tuberculosis when administering rituximab versus anti-TNF $\alpha$ medicines is very low; the most serious side effects include interstitial lung damage occurring in 0.01 to $0.03 \%$ of treated patients (10). The interstitial lung damage may be fatal. A case with pulmonary involvement associated with rituximab therapy imitating milliary tuberculosis was described (10). Rituximab affects the results of IGRA tests. 


\section{Anti CD 52 treatment}

CD52 is a glycoprotein, which is encoded by the CD52 gene in humans. CD52 is found on the surface of mature lymphocytes, but not on the surface of their stem cells. It is also found on the surface of monocytes and dendritic cells. It is a peptide composed of 12 aminoacids anchored on the cell surface through glycosylphosphatidylinositol chain. It has anti-adhesive properties. CD52 is detected in some lymphomas.

Monoclonal antibody against CD52 (alemtuzumab) is used to treat chronic lymphocytic leukaemia and is currently tested in the treatment of multiple sclerosis. The risk of developing tuberculosis compared to anti-TNF $\alpha$ is low but higher than in rituximab. The individual patient risk depends on the epidemiological situation in the region (3).

\section{Anti CD 80, CD 86 treatment}

CD80 or also B7-1 is a protein expressed on the surface of activated $\mathrm{B}$-lymphocytes and monocytes, which provides the necessary costimulatory signal for activation and survival of T-lymphocytes. Its ligands are CD28 (for autoregulation and intercellular associations) and CTLA-4 (for deceleration of regulation and cell dissociation). CD80 together with CD86 stimulate T-lymphocytes. CD86 or also B7-2 is a protein found on the surface of antigen-presenting cells, which provides a costimulatory signal to T-lymphocytes. It has the same ligands as CD80. CD86 gene encodes a membrane protein, which is a member of the immunoglobulin superfamily.

Abatacept is a fusion protein consisting of the IgG1 Fc region and the extracellular domain CTLA-4. It is indicated for the treatment of rheumatoid arthritis, it should not be administered concomitantly with anti-TNF $\alpha$ and anakinra. Abatacept is currently tested in focal segmental glomerulosclerosis and ulcerative colitis. The risk of tuberculosis in patients treated with abatacept is low (1).

\section{Anti TNF $\alpha$ treatment}

Tumour necrosis factor alpha (TNF $\alpha)$ is a cytokine first described in 1968 by Granger and Ruddle as a cytotoxic factor produced by activated lymphocytes. Activated macrophages, CD4+ T-lymphocytes, NK cells and neuronal cells also produce this pleiotropic cytokine. It is a protein comprising 212 amino acids, whose gene is located on chromosome $6 \mathrm{p} 21.3$. TNF $\alpha$ binds to two types of receptors, TNF-RI (CD120a, p55/60) and TNF-RII (CD120b, p75/80). TNF-RI is found on many cells, responds to membrane-bound and soluble TNF $\alpha$. TNF-RII is located only on immune cells and responds only to membrane bound TNF $\alpha$. TNF $\alpha$ is vital for the inflammatory and immune response; it is essential e.g. for granuloma formation. A TNF $\alpha$ knockout mouse is not capable of forming granulomas.

The following five anti-TNF $\alpha$ medicines, infliximab, etanercept, adalimumab, certolizumab pegol and golimumab are currently available for clinical practice. Indications of infliximab include rheumatoid arthritis, Crohn's disease, psoriatic arthritis, ankylosing spondylitis and some other diseases. Adalimumab, golimumab and certolizumab pegol are administered in the same indications. Etanercept is indicated for rheumatoid arthritis, ankylosing spondylitis, psoriatic arthropathy and juvenile idiopathic arthritis.

\section{Anti IL-6 treatment}

Interleukin 6 (IL-6) is a cytokine, involved in the inflammation pathogenesis. It is produced by stimulated T-lymphocytes and macrophages. IL-6 may also act as an anti-inflammatory agent through blockage of the effects of TNF $\alpha$ and IL-1 and activation of the IL-1ra and IL-10. IL-6 induces fever and production of acute phase proteins by hepatocytes. IL- 6 crosses the blood brain barrier and induces synthesis of PGE2 in the hypothalamus leading to an increase in the body temperature. IL-6 induces a release of energy from muscles and adipose tissue, which leads to an increase in the body temperature. IL-6 is produced by adipocytes and is probably the cause of the increase of CRP in obese individuals. IL- 6 binds to the receptor complex on the cell surface, which comprises the binding site - IL-6R $\alpha$ chain (CD126) and signal transmitting component gp130 (CD130). Signal transduction is mediated by transcription factors, the Janus kinase (JAK) and signal carriers and activators of transcription (STAT). Other cytokines, in which the signal is mediated by receptors containing gp130 like interleukin 11 (IL-11), interleukin 27 (IL-27), ciliary neurotrophic factor (CNTF), cardiotrophin-1 (CT-1), cardiotrophin-like cytokine and more factors called IL-6 like or gp130 utilising cytokines. In addition to the cell membrane bound receptor for IL- 6 there is also a soluble form (sIL-6R). IL-6 stimulates inflammatory and autoimmune processes in a number of diseases, e.g. in diabetes, atherosclerosis, depression, Alzheimer's disease, systemic lupus erythematosus, multiple myeloma, prostate cancer, Behcet's disease and rheumatoid arthritis.

Tocilizumab is a monoclonal antibody blocking the IL6 receptor. It does not suppress the production of IFN $\gamma$. Tocilizumab is indicated in rheumatoid arthritis, juvenile idiopathic arthritis and Castleman"s disease. Its risk for the development of tuberculosis is lower compared to the anti TNF $\alpha$ agents. LTBI screening is recommended.

\section{Anti IL-1 treatment}

IL-1 family is a group of 11 cytokines that induce and regulate inflammatory responses of the body. IL- $1 \alpha$ and IL- $1 \beta$ are the main representatives because they were described and studied first. Their natural antagonist IL-1Ra. IL-1 $\alpha$, IL-1 $\beta$ and IL-1Ra bind to IL-1 receptor (IL-1R). All 11 cytokines except for IL-1Ra are synthesized as precursors that are cleaved by proteolysis to active proteins. IL-1 is produced by macrophages, monocytes, fibroblasts and dendritic cells; it is also expressed by B-lymphocytes, NK cells and epithelial cells. IL-1 is an important part of the inflammatory response of the body against infection. IL-1 group increases the expression of adhesion molecules on endothelial cells, thereby enabling migration of immunocompetent cells to the infection site. IL-1 group affects hypothalamus activity, e.g. the thermoregulatory centre, which leads to increase in body temperature. IL-1 is 
an endogenous pyrogen. In addition to fever, IL-1 also induces hyperalgesia, vasodilatation and hypotension. IL- $1 \alpha$ is synthesized as a precursor that is stored in the cytoplasm of cells of the mesenchymal and epithelial origin. Monocytes and macrophages do not have IL-1 $\alpha$ precursors in their cytoplasm; they synthetize this cytokine de novo. IL-1 $\alpha$ precursor is cleaved by a calciumactivated protease to the active $17-\mathrm{kDa}$ protein with the release of $16-\mathrm{kDa}$ N-terminal protein (ppIL-1 $\alpha$ ), which acts as a transcription factor. The precursor form of IL- $1 \alpha$, which contains both the Nterminal and C-terminal receptor domain, acts as a DAMP molecule (damage-associated molecular pattern). These molecules, also known as alarmins, are recognized by cells of innate immunity and act as danger signals to the immune system. Cellular stress induces infection, trauma, ischemia, hypoxia, acidosis and complement lysis. Inflammatory response in the absence of infection, e.g. after ischemia, depends only on the signal mediated by binding IL- $1 \alpha$ to IL-1R, not by the toll-like receptors (TLR). IL- $1 \beta$ is synthesized as a precursor molecule, but only after stimulation of cells. Its expression is induced by the transcription factor NF- $\kappa \mathrm{B}$ following stimulation of innate immunity by alarmins. Following the exposure to LPS, which binds to TLR-4 by monocytes/macrophages and dendritic cells, are synthesized the IL-1 $\beta$ and IL-18 precursors. Both precursors are cleaved by caspase-1.

Anakinra is a registered medicine for treatment of rheumatoid arthritis. It is an antagonist of interleukin-1 receptor (IL-1Ra). Anakinra blocks the biological activity of IL-1, inflammation and cartilage destruction in patients with RA. In patients with RA, concentrations of IL1-Ra in the synovial membrane and synovial fluid were detected that were insufficient to neutralize the effect of increased concentrations of IL-1. Anakinra is also currently tested in indolent or smouldering myeloma with a high risk of progression to multiple myeloma. Anakinra should not be used in combination with TNF $\alpha$ inhibitors such as etanercept, infliximab or adalimumab. The side effects include frequent upper respiratory infection ( $13 \%$ of patients), sinusitis ( $7 \%$ of patients), and flu-like syndrome ( $6 \%$ of patients). Pneumonia and tuberculosis are reported as rare complications.

\section{Anti IL-12, IL-23 treatment}

Macrophages, dendritic cells and B-lymphoblastoid elements produce IL-12. It is a heterodimer coded by two genes. IL-12 stimulates growth and differentiation of T-lymphocytes, increases cytotoxic activity of NK cells and stimulates production of TNF $\alpha$ and IFN $\gamma$ by T-lymphocytes, NK cells and cytotoxic CD8+ Tlymphocytes. IL-12 is important in protective immunity against intracellular pathogens (tuberculosis, mycobacteriosis, salmonella and others). Furthermore, it plays an important role in autoimmunity. IL-23 is a heterodimer consisting of IL-12p40 and IL-23p19 chains. It is a cytokine, which plays a role in the pathogenesis of inflammation.

Ustekinumab is a human monoclonal antibody against IL12 and IL-23. It is indicated in the treatment of psoriatic arthritis and some severe forms of psoriasis. Its effect is tested in multiple sclerosis and sarcoidosis. Cases of transition of LTB into active tuberculosis during its administration were published in literature. Experience with this medicine is still short-term and the need for examination of the patient depends on assessment of the individual risk (11).

\section{Risk of tuberculosis in biological therapy}

Tuberculosis is an infectious disease caused by Mycobacterium tuberculosis complex. It can be characterized as granulomatous inflammation with a tendency to necrosis. Patients treated with anti-TNF $\alpha$ have 1.6 to 25.1 times higher risk of tuberculosis as indicated in literature $(4,6,12,13,18)$. The risk of tuberculosis depends on the prevalence of tuberculosis in the region and the type of medicine used. Currently, in clinical practice we use the following anti-TNF $\alpha$ agents: infliximab, etanercept, adalimumab, certolizumab pegol and golimumab. Indications of infliximab include rheumatoid arthritis, Crohn's disease, psoriatic arthritis, ankylosing spondylitis and some other diseases. Adalimumab, golimumab and certolizumab pegol are administered in the same indications. Etanercept is indicated for rheumatoid arthritis, ankylosing spondylitis, psoriatic arthropathy and juvenile idiopathic arthritis. From the biological medicines used, the lowest risk of tuberculosis is connected with etanercept, however examination of patients before starting biological therapy are recommended for all medicines administered. In a British study, which analysed the risk of individual medicines among 10,712 patients, it was found that infliximab has 3 times higher risk of developing tuberculosis than etanercept (6). In this set, tuberculosis developed in the total of 40 patients; there were 15 cases of pulmonary tuberculosis and 25 cases of extrapulmonary tuberculosis.

A pneumologist during the examination of patients who are scheduled to initiate biological therapy investigates and assess the medical history, clinical examination, chest X-ray, tuberculin test (MxII) results and the results of IGRA tests (QuantiFERON-TB Gold, T-SPOT.TB). In children under 2 years of age, tuberculin test is preferred. In adults, IGRA tests are considered to be more sensitive and specific; the results are not influenced by previous BCG vaccination. Most national guidelines recommend use of both tuberculin skin test and IGRA test. In the case of indicated chemoprophylaxis or anti-tuberculous treatment, the patient is monitored by a pneumologist.

\section{Chemoprevention}

Chemoprevention includes INH administration at the dose of $5 \mathrm{mg} / \mathrm{kg}$, along with pyridoxine for 6-9 months. Any contact or infected individual takes the chemoprophylaxis only once. During chemoprophylaxis it is necessary to monitor liver function tests in monthly intervals. In exceptional cases, instead of INH it is possible to administer RMP at the dose of $10 \mathrm{mg} / \mathrm{kg}$ for 4 months (neurotoxicity of INH, INH-resistant strain) or a combination of $\mathrm{RMP}+\mathrm{INH}$ daily for 3 months. In patients diagnosed with active tuberculosis, pneumologist initiates the therapy - combination of four antituberculotics. Treatment with antituberculotics is repeated in the case of tuberculosis relapse. 


\section{Practical recommendations}

A pneumologist makes a decision according to the following criteria:

Situation 1: No family and personal history of tuberculosis, nor any current contact with TB. The patient is asymptomatic in terms of TB with normal findings on chest X-ray. Tuberculin skin test (MxII) is negative (induration $0-5 \mathrm{~mm}$ ), QuantiFERON-TB Gold or T-SPOT TB are also negative. In this case, it is possible to initiate biological treatment; monitor the patient by a pneumophtiseologist every 6 months during the first year, then once a year.

Situation 2: No family and personal history of tuberculosis, nor any current contact with TB. Asymptomatic in terms of TB; normal findings on chest X-ray. The tuberculin test (MxII) is positive (6-15 $\mathrm{mm}$ ), QuantiFERON-TB Gold or T-SPOT.TB negative. With these findings, we do not assume presence of latent tuberculosis infection, a positive tuberculin skin test might be due to the BCG vaccination. In thiscase, it ispossible toinitiate biological therapy; monitor the patient by a pneumologist every 6 months in the first year, then once yearly.

Situation 3: No family and personal history of tuberculosis, nor any current contact with TB in the patient. The patient is asymptomatic in terms of TB with normal findings on chest X-ray. Tuberculin skin test (MxII) is hyperreactive (induration above 15 $\mathrm{mm}$ ) and IGRA tests (QuantiFERON-TB Gold or T-SPOT.TB) are negative or uncertain. This situation may occur rarely. Given that according to the MxII results there is hyperreactivity, we diagnose the patient with latent tuberculosis. Preventive therapy with INH 5 $\mathrm{mg} / \mathrm{kg}$ for six months is indicated; biological therapy may be initiated after two months of preventive therapy.

Situation 4: No family and personal history of tuberculosis, nor any current contact with TB in the patient. Asymptomatic in terms of TB, normal findings on chest X-ray. Tuberculin skin test (MxII) is negative ( $0-5 \mathrm{~mm}$ induration), QuantiFERON-TB Gold or T-SPOT.TB are positive. There is evidence of latent tuberculosis infection. The patient is indicated for preventive therapy with INH $5 \mathrm{mg} / \mathrm{kg}$ for 6 months; biological therapy may be initiated after 2 months of preventive therapy.

Situation 5: No family and personal history of tuberculosis, nor any current contact with TB in the patient. Asymptomatic in terms of TB; normal findings on chest X-ray or small fibrotic lesion. Tuberculin skin test (MxII) is above $15 \mathrm{~mm}$ - hyperreactive, QuantiFERON-TB Gold or T-SPOT.TB are positive. The patient is indicated for preventive therapy with INH $5 \mathrm{mg} / \mathrm{kg}$ for 6 months; biological therapy may be initiated after 2 months of preventive therapy.

Situation 6: No family and personal history of tuberculosis, current contact with infectious TB. Normal findings on chest Xray or small fibrotic lesion. Tuberculin skin test (MxII) is above $15 \mathrm{~mm}$ - hyperreactive, QuantiFERON-TB Gold or T-SPOT.TB are positive. The patient is indicated for preventive therapy with INH $5 \mathrm{mg} / \mathrm{kg}$ for 6 months; biological therapy may be initiated after 2 months of preventive therapy.

Situation 7: The patient is diagnosed with active tuberculosis. A pneumologist initiates the treatment with antituberculotics. Biological treatment is possible after the completion of the chemotherapy and negative MTB cultures.

\section{References}

1. Alten R, Kaine J, Keystone $\mathbf{E}$ et al. Long-term safety of subcutaneous abatacept in rheumatoid arthritis: integrated analysis of clinical trial data representing more than four years of treatment. Arthritis Rhematol 2014; 66 (8): 1987-1997.

2. Amano K. Tocilizumab. Nihon Rinsho 2013; 71 (7): 1238-1242.

3. Au WY, Leung AY, Tse EVV et al. High incidence of tuberculosis after alemtuzumab treatment in Hong Kong Chinese patients. Leuk Res 2008; 32 (5): 547-551.

4. Balbir-Gurman, A, Lidgi M, Elkayam O. Update of the guidelines of the Israeli Association of Rheumatology for the prevention of tuberculosis in patients treated with TNF-alpha blockers. Harefuah 2014; 153 (6): 359-361.

5. Cantini F, Niccoli L, Goletti D. Tuberculosis risk in patients treated with non-anti-tumor necrosis factor- $\alpha(\mathrm{TNF}-\alpha)$ targeted biologicals and recently licensed TNF- $\alpha$ inhibitors: data from clinical trials and national registries. $J$ Rheumatol 2014; Suppl 91: 56-64.

6. Dixon WG, Hyrich KL, Watson KD et al. Drug-specific risk of tuberculosis in patients with rheumatoid arthritis treated with anti-TNF therapy: results from the British Society for Rheumatology Biologis Register (BSRBR). Ann Rheum Dis 2010; 69 (3): 522-528.

7. De Keyser F. Choice of biologic therapy for patients with rheumatoid arthritis: The infection perspective. Curr Rhematol Rev 2011; 7 (1): 77-87.

8. Edwards J, Szczepanski L, Szechinski J, Filipowicz-Sosnowska A et al. Efficacy of B-cell-targeted therapy with rituximab in patients with rheumatoid arthritis. N Engl J Med 2004; 350 (25): 2572-2581

9. Chen, YM, Chen, HH, Lai, KL et al. The effects of rituximab therapy on released interferon- $\gamma$ levels in the QuantiFERON assay among rheumatoid arthritis patients with different status of Mycobacterium tuberculosis infection. Rheumatology (Oxford) 2013; 52 (4): 697-704.

10. Chih-Wei Y, Wei-Chih L, Chih-Yen T et al. Rituximab-induced pneumonitis mimicking miliary tuberculosis. Eur Resp Rev 2013; 22 (3): 587-588.

11. Leonardi CL, Kimball AB, Papp KA et al. Efficacy and safety of ustekinumab, a human interleukin-12/23 monoclonal antibody, in patients with psoriasis: 76-week results from a randomised, double-blind, placebocontrolled trial (PHOENIX 1). Lancet 2008; 371 (9625): 1665-1674.

12. Ruderman EM. Overview of safety of non-biologic and biologic DMARDs. Rheumatology (Oxford) 2012; 51 (Suppl 6): 37-43.

13. Rigal E, Gatenault P, Lebranchu Y, Hoarau C. Therapeutic monoclonal antibodies: update on the risk of opportunistic infections. Med Sci (Paris) 2009; 12 (12): 1135-1140.

14. Saini KS, Azim HAJr, Cocorocchio E, Vanazzi A et al. Rituximab in Hodgkin lymphoma: Is the target always a hit? Cancer Treat Rev 2011; 37 (5): 385-390.

15. Schiff M, Weinblatt ME, Valente $\mathbf{R}$ et al. Head to head comparison of subcutaneous abatacept versus adalimumab for rheumatoid arthritis: twoyear efficacy and safety findings from AMPLE trial. Ann Rheum Dis 73; 2014 (1): 86-94.

16. Segal BM, Constantinescu CS, Raychaudhuri A, Kim L, Fidelus-Gort R, Kasper LH. Repeated subcutaneous injections of IL12/23 p40 neutralising antibody, ustekinumab, in patients with relapsing-remitting multiple sclerosis: a phase II, double-blind, placebo-controlled, randomised, dose-ranging study. Lancet Neurol 2008; 7 (9): 796-804.

17. Souto A, Maneiro JR, Salgado E et al. Risk of tuberculosis in patients with chronic immune-mediated inflammatory diseases treated with biologicals and tofacitinib: a systematic review and meta-analysis of randomized controlled trials and long-term extension studies. Rheumatology (Oxford) 2014; 53 (10): 1872-1885.

18. van der Have $\mathrm{M}$, Oldenburg B, Fidder $\mathrm{HH}$ et al. Optimizing screening for tuberculosis and hepatitis B prior to starting tumor necrosis factor- $\alpha$ inhibitors in Crohn's disease. Dig Dis Sci 2014; 59 (3): 554-563.

Received April 24, 2015. Accepted May 20, 2015. 